\title{
A Model For Residential Adoption of Photovoltaic Systems
}

\author{
Thesis by \\ Anish Agarwal \\ In Partial Fulfillment of the Requirements \\ for the Degree of \\ Master of Science
}

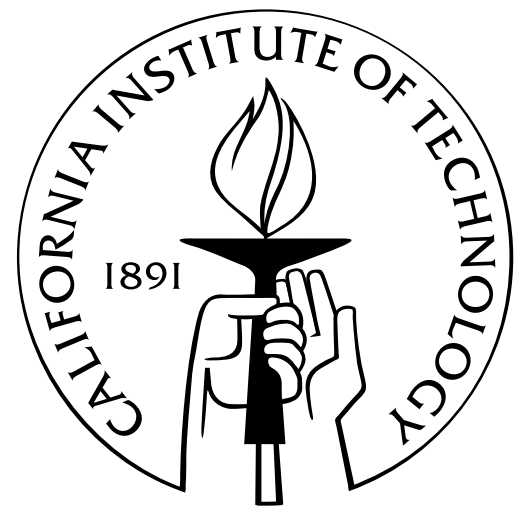

California Institute of Technology

Pasadena, California

2015

(Submitted March 25, 2015) 
(C) 2015

Anish Agarwal

All Rights Reserved 


\section{Acknowledgements}

First and foremost, I would like to thank my adviser Prof. Mani Chandy. He took a chance on me even though I was by no means qualified. In addition, there were moments along the way when things looked quite stark and Mani's support was invaluable in me getting through it. For this, I will always be in his debt. He is one of a handful of researchers I have met that manages to conduct research that has both academic merit and yet a large societal impact. Still, the most valuable thing I have learned from Mani is how to be a good person.

I would also really like to thank Prof. Adam Wierman. I always looked forward to our Fridays afternoon meetings. I spent days banging my head against a wall on how to solve a problem but inevitably left his office at the end of the week with a clear sense of direction for the next. Over the past two years (along with innumerable beers at the Ath, a triathlon and a round of golf), Adam has gone from simply being a mentor to a friend.

Also, I would really like to thank my last co-advisor (yes, I had three) Prof. Steven Low. From him I learned what it really means to have a single-minded devotion to an idea and the work ethic plus tenacity it takes to make it happen.

I would also especially like to thank my main collaborator and partner-in-crime, Desmond Cai. His work was the basis for this thesis and this work would not have been even close to possible if not for his patient and insightful guidance through the process. I will miss our frequent and leisurely mid-afternoon coffee breaks. I learned as much from just chatting casually with him as I did in all my classes. I would also like to thank Niangjun Chen and 
Subhonmesh Bose for giving me a chance to work with them on a variety of projects. My experiences with them have made me a significantly better researcher. Lastly, I want to thank all the members of RSRG. It really is a one-of-a-kind group in both breadth and depth. It was one of the most intellectually stimulating experiences I've had listening to everyone speak about their work in our group lunches.

And finally, I thank my parents. When I feel I am losing my way, I hear my father's voice in my head nudging me back onto the correct path. When I feel down, I hear my mother's voice in my heart giving me the strength to push through. For this, I will be forever grateful. 


\section{Abstract}

The rapid rise in the residential photo voltaic (PV) adoptions in the past half decade has created a need in the electricity industry for a widely-accessible model that estimates $\mathrm{PV}$ adoption based on a combination of different business and policy decisions. This work analyzes historical adoption patterns and finds fiscal savings to be the single most important factor in PV adoption, with significantly greater predictive power compared to all other socioeconomic factors including income and education. We can create an application available on Google App Engine (GAE) based on our findings that allows all stakeholders including policymakers, power system researchers and regulators to study the complex and coupled relationship between PV adoption, utility economics and grid sustainability. The application allows users to experiment with different customer demographics, tier structures and subsidies, hence allowing them to tailor the application to the geographic region they are studying. This study then demonstrates the different type of analyses possible with the application by studying the relative impact of different policies regarding tier structures, fixed charges and PV prices on PV adoption. 


\section{Contents}

Acknowledgements

Abstract $\quad$ v

1 Introduction 1

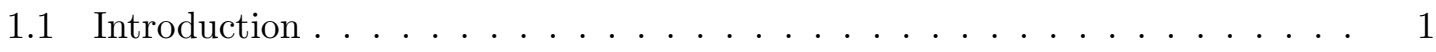

2 Data Analysis $\quad 4$

2.1 Data Analysis . . . . . . . . . . . . . . . . . . . . . 4

2.1.1 Data on residential customers . . . . . . . . . . . . . . 4

2.1 .2 Customers' savings from solar PV . . . . . . . . . . . 6

2.1.3 Methodology for variance tests . . . . . . . . . . . . . 7

2.1.4 Results of variance tests . . . . . . . . . . . . . . . 9

3 Adoption Model $\quad 11$

3.1 Adoption Model . . . . . . . . . . . . . . . . . 11

3.1 .1 Diffusion model for adoption . . . . . . . . . . . 12

3.1 .2 Fitting diffusion parameters . . . . . . . . . . . . . . 12

4 Simulation Tool $\quad 14$

4.1 Simulation Tool . . . . . . . . . . . . . . . . . . . . . . . . 14

4.2 Simulation Runs . . . . . . . . . . . . . . . . . . 15 
vii

4.2.1 Description of different rates and policies tested . . . . . . . 15

4.2 .2 Results of simulations . . . . . . . . . . . . . . . 17

5 Conclusion $\quad 19$

5.1 Conclusion . . . . . . . . . . . . . . . . . . . . . 19

5.1 .1 Strengths of Model . . . . . . . . . . . . . . . . . . . . . . 19

5.1 .2 Weaknesses of Model . . . . . . . . . . . . . . . . . . . 20

5.1 .3 Applications . . . . . . . . . . . . . . . . . 21

5.1 .4 Future steps . . . . . . . . . . . . . . . . . . 21

$\begin{array}{ll}\text { Bibliography } & 22\end{array}$ 


\section{Chapter 1}

\section{Introduction}

\section{$1.1 \quad$ Introduction}

There has been a rapid rise in the number of residential solar photo voltaic (PV) adoptions in the past half decade. Indeed, recent policies and incentives on solar energy [13, 19] reflect that this increased popularity in $\mathrm{PV}$ is a product both of consumer preference and a push by governmental institutions to move towards a carbon free society. This change is not without major challenges - it will require significant upgrades to the grid as it was not engineered with the variability and intermittency of solar energy in mind [20, 29]. In addition to engineering hurdles, we need to be mindful of the socioeconomic impacts of large scale adoption which most commonly come in the form of subsidies to high income customers $[5,10,14,1]$. Under the widely used net metering rate structures, residential customers only pay for the net amount of monthly energy used. However since fixed infrastructure upkeep costs are typically recovered through these net metering charges, PV customers end up contributing less to infrastructure costs than customers that do not own PV even though they still utilize grid infrastructure to get electricity. Since higher income customers use more electricity and are charged at a higher rate for every marginal wattage consumed, they have a much higher incentive to adopt PV.

Hence there is a strong need for a model that predicts distributed PV adoption based 
on the wide variety of business and policy decisions that can be made. Foe example regulators need such a tool to asses the societal impact, both intentional and unintended, of PV adoption and design rate structures that can hopefully lessen potentially undesirable consequences. On the other hand, utility company executives need to make timely and cost-effective infrastructure upgrades based on foretasted demand for PV.

In this paper, a model is proposed to forecast uptake of distributed solar PV, that can be used to study rate structures, monetary incentives for PV, and utility infrastructure upgrades. We make the following three contributions:

1. We analyze historical data on PV adoption and show monetary savings is the most significant factor driving adoption (superseding all socioeconomic factors in importance).

2. Using the insights gathered from our data analysis, we propose a model for PV uptake. Our model is based on an extension of the well-established Bass diffusion model for technology adoption. Our validation tests demonstrate that the proposed model gives fairly accurate predictions of solar PV uptake.

3. We present examples of using our model to study various rate structures and policy decisions.

Prior studies on understanding the drivers of PV adoption among residential customers have focused on the importance of socioeconomic factors, customer demographics and social contagion $[13,16,15,3]$. In particular, a recent study showed that income is the most strongly correlated with adoption [13]. To our knowledge, there has been no prior work on the impact of financial savings from PV on adoption rates. The latter is important as financial savings could be the underlying driver of adoption and simply correlated with income. In addition, prior attempts to build a diffusion based adoption model have not factored in the financial savings when fitting model parameters with historical data $[5,18$, $4,21]$. 
There has also been relatively little work on studying the impact of financial savings through PV adoption on the utility "death spiral" $[5,17]$. The "death spiral" refers to the positive feedback effect created when the highest consuming customers adopt PV and thus pay less to utilities. This in turn causes utilities to raise rates so that the grid can be maintained, which incentivizes even more customers to adopt PV. We extend a previous study on the utility "death spiral" [5] by analyzing historical data and explicitly factoring in financial savings when studying this feedback effect. 


\section{Chapter 2}

\section{Data Analysis}

\subsection{Data Analysis}

In this section, we present our analysis of historical PV adoption.

\subsubsection{Data on residential customers}

Our residential customer dataset describes the consumption patterns and socioeconomic backgrounds of approximately 4 million households in Southern California Edison (SCE). The dataset includes the following features for each household:

1. Nielsen PRIZM cluster [12];

2. Monthly consumption from July 2012 to June 2013 in kWh;

3. Rate schedule during each of those months;

4. Climate zone;

5. Size and date of installation of PV system (if one is installed).

The zip code, monthly consumption, and rate schedule of every household was made available to us through a non-disclosure agreement with SCE. From the zip code, we derived 
each household's PRIZM segment and climate zone from publicly available sources [7, 23]. We also obtained the size and date of all residential PV installations from SCE.

Nielsen PRIZM cluster: PRIZM is a widely used customer segmentation system which provides information about a household's socioeconomic background. It places each household into one of sixty-six clusters, and each cluster uniquely maps customers to a specific combination of seven features: (i) income level, (ii) education attainment, (iii) employment type, (iv) home ownership, (v) location, (vi) age range, and (vii) family type.

Each features is comprised of between five to seven categories. For example, income levels are partitioned into seven categories ranging from households earning less than $\$ 10,000$ per annum to those earning more than $\$ 100,000$ per annum, the education attainment feature is comprised of five categories that range from attending high school to attaining a post graduate degree. Detailed descriptions of all of the features and the sixty-six PRIZM clusters can be found in [12].

Rate schedule: SCE currently offers 18 distinct residential rate schedules. However, about $92 \%$ of customers subscribe to either Schedule D or Schedule D-CARE. Only customers that subscribed to either of these two rates were considered in our analysis. Both rate schedules have block-inclining rates; price for each incremental $\mathrm{kWh}$ of electricity consumed increases. Schedule D-CARE is a subsidized rate schedule with a discount of about $20 \%$ off Schedule D rates [27].

Notable omissions in our analysis were customers that were under time-of-use (TOU) rates and the summer-discount-plan (SDP) [27]. These omissions will not have a significant affect on our analysis as these customers made up less than 10\% of all customers or $30 \%$ of all PV adopters. Furthermore, a household's decision to switch from Schedule D to TOU or SDP is primarily to lower their electricity bill. Our analysis already indicates that monetary savings is the most significant factor driving adoption and so including TOU and SDP customers should further corroborate our results. 


\subsubsection{Customers' savings from solar PV}

The purchase of PV is often accompanied by a significant fiscal incentive. There are a variety of approaches to measure the monetary incentives of PV since these savings are accrued over the lifetime of the PV system (which is typically at least 20 years). In this study, we focus on the net present value (NPV) of the total expected monthly savings over the 20-year period immediately after a household installs solar PV:

$$
\text { Savings }=\sum_{y=1}^{20} \delta^{y-1}\left(\sum_{m=1}^{12}\left(b_{m, y}-b_{m, y}^{\prime}\right)\right)-\text { CostOfPV }
$$

where $b_{m, y}$ is the expected utility bill in month $m$ of year $y$ if the customer does not adopt $\mathrm{PV}$ (in $\mathrm{kWh}), b_{m, y}^{\prime}$ is the expected utility bill in that month if the customer adopts PV (in $\mathrm{kWh}$ ), $\delta=0.95$ is the annual discount factor (assumed to be $5 \%$ ), and CostOfPV is the NPV of the costs of the PV system.

Households that have PV: For households that have PV, we approximate their expected savings at the time of installation using the dataset. We use the household's climate zone and irradiation data to estimate the amount of electricity that would be generated by their PV system [6]. We assume that a household's annual electricity usage is identical to what it was between July 2012 and June 2013 and that it subscribes to net-metering. Then, we calculate the expected future utility bills based on the electricity rates at the time of installation. $^{1}$

We estimate the NPV of the purchasing costs using the size and installation date of the PV systems. In particular, we obtain PV prices (in $\$ / \mathrm{kW}$ ) paid by residential customers in SCE between 2007 and 2012 from the California Solar Initiative (CSI) database [9] and fitted a linear model to the data to estimate PV prices based on the installation date. Finally, we adjusted these costs for the $30 \%$ federal tax rebates and CSI rebates. Note

\footnotetext{
${ }^{1}$ Prior studies have found that high renewable penetrations can drive substantial changes in residential retail rates [11]. However, our assumption is still reasonable as PV adopters typically receive estimates on future bill savings (from solar companies) based on existing residential retail rates.
} 
7

\begin{tabular}{lllll}
\hline & $\begin{array}{l}\text { Average } \\
\text { Monthly } \\
\text { Usage }(\mathrm{kWh})\end{array}$ & $\begin{array}{l}\text { PV System } \\
\text { Size }(\mathrm{kW})\end{array}$ & $\begin{array}{l}\text { Savings: } \\
\text { Non-CARE } \\
(\$)\end{array}$ & $\begin{array}{l}\text { Savings: } \\
\text { CARE }(\$)\end{array}$ \\
\hline Bin 1 & 130 & 1.0 & -637.6 & -1961.1 \\
Bin 2 & 300 & 2.0 & -1237.136 & -3895.2 \\
Bin 3 & 500 & 2.0 & 1947.0 & -1253.0 \\
Bin 4 & 690 & 3.0 & 7594.5 & 1624.2 \\
Bin 5 & 890 & 3.0 & 11791.9 & 3974.5 \\
Bin 6 & 1300 & 6.0 & 24835.9 & 7944.8 \\
\hline
\end{tabular}

Table 2.1: Estimated savings for households that do not have solar PV segmented by usage category.

that our model does not differentiate between owned and leased systems as these data are not available. However, prior studies have found that buyers and leasers do not necessarily differ significantly along socio-demographic variables[26].

Households that do not have solar PV: To analyze monetary savings as a driver of solar PV adoption, we also need to estimate the savings that households who have not installed solar PV would be able to obtain by adopting PV. For instance, if all households who have not installed solar PV have significantly less savings than the existing owners of solar PV, it indicates that monetary savings is an important driver of solar PV adoption. However, there is no information on the size and costs of the PV systems these households would install. We assume they would adopt the same PV system sizes as the households with similar usage and who have adopted PV. Finally, we computed the costs of PV based on PV prices in 2012. The system sizes and estimated savings are shown in Table 2.1.

\subsubsection{Methodology for variance tests}

We identify the customer features that are most correlated with solar PV adoption by analyzing the variance of adoption along different features. The features that reduce the variance the most can be interpreted as the features that are most crucial for predicting solar PV adoption. We call this methodology variance testing. 
Let $N$ denote the total number of customers and $\mathbf{x}=\left(x_{1}, x_{2}, \ldots, x_{N}\right)$ denote the adoption data points such that $x_{i}=1$ if the $i$ th customer has adopted solar PV and $x_{i}=0$ otherwise. Let $\mathcal{B}=\left\{B_{1}, B_{2}, \ldots, B_{K}\right\}$ denote a partition of the customers $\{1,2, \ldots, N\}$ into $K$ bins. We define the variance of adoption under partition $\mathcal{B}$ as:

$$
\operatorname{Variance}(\mathcal{B})=\frac{1}{N-1} \sum_{k=1}^{K} \sum_{i \in B_{k}}\left(x_{i}-M_{k}\right)^{2}
$$

where:

$$
M_{k}=\frac{1}{\left|B_{k}\right|} \sum_{i \in B_{k}} x_{i}
$$

is the fraction of adopters in set $B_{k}$.

The term $\sum_{i \in B_{k}}\left(x_{i}-M_{k}\right)^{2}$ is the scaled sample variance of adoption in set $B_{k}$. Hence, Variance $(\mathcal{B})$ can be interpreted as the weighted-sum of the sample variances in the partitions in $\mathcal{B}$ (weighted by the relative sizes of the partitions). Notice that the sample variance of adoption in a partition equals zero when all customers in that bin adopts $\left(M_{k}=1\right)$ or do not adopt $\left(M_{k}=0\right)$. Moreover, this sample variance is maximized when exactly half of the customers in that bin adopts $\left(M_{k}=1 / 2\right)$. Hence, one could interpret Variance $(\mathcal{B})$ as a measure of how well the partition segments customers into categories with different likelihoods of adoption. A smaller variance implies that the partition is relatively better at segmenting the customer base into those with higher likelihood to adopt and vice-versa.

Hence, our goal is to identify a meaningful system of partitions which provides the most drop in adoption variance. As an extreme example, we could partition the customers such that every customer who has PV are in one bin and those who do not are in another bin, and get $\operatorname{Variance}(\mathcal{B})=0$. However, this approach provides no insight into what factors are correlated with adoption. For this study, we analyze the variance under the partitions defined by the socioeconomic features in the PRIZM clusters and monetary savings from 
9

\begin{tabular}{lll}
\hline Feature & Variance & Change \\
\hline Savings & 0.018985933 & -0.000426144 \\
Income Level & 0.019345964 & -0.000066113 \\
Education Attainment & 0.019355716 & -0.000056361 \\
Employment Type & 0.01935733 & -0.000054747 \\
Location & 0.019358202 & -0.000053875 \\
Home Ownership & 0.01937226 & -0.000039817 \\
Age Range & 0.01937678 & -0.000035297 \\
Family Type & 0.019377073 & -0.000035004 \\
No Segmentation & 0.019412077 & - \\
\hline
\end{tabular}

Table 2.2: Adoption variance without segmentation and with segmentation along a single feature. The last column shows the change in adoption variance between the segmented and unsegmented cases.

\begin{tabular}{ll}
\hline Feature & Variance \\
\hline Savings + Income Level & 0.018955343 \\
Savings + Location & 0.018956756 \\
Savings + Home Ownership & 0.018964283 \\
Savings + Employment Type & 0.018965506 \\
Savings + Family Type & 0.018967814 \\
Savings + Education Attainment & 0.018968473 \\
Savings + Age Range & 0.018972375 \\
Savings & 0.018985933 \\
\hline
\end{tabular}

Table 2.3: Adoption variance with segmentation by savings and some other feature.

adoption. For socioeconomic features, we use the bins defined by the PRIZM clusters. For savings, we partition customers into eight equally-spaced bins (from $\$ 0$ to $\$ 30,000$ ).

\subsubsection{Results of variance tests}

Table 2.2 shows the results of the variance tests. To provide a benchmark, we also compute the adoption variance of the original data without any segmentation. We see that economic savings from solar PV adoption causes significantly larger drop in adoption variance compared to any other feature (about 7 times the drop in variance under the next most important feature). The next three factors in order of importance are income, edu- 
cation and employment, which corroborates the findings of many prior studies that only investigated socioeconomic factors without considering fiscal incentives $[13,16,15]$.

Next, we calculate the additional variance drop if we include another feature along with savings. That is, each bin is a unique combination of a savings level and a category from the other feature. The results are shown in Table 2.3. Among the non-savings features, income provides the largest drop in variance. However, this drop is still small compared to the original drop in variance provided by economic savings alone - only $7.17 \%$ of that provided by economic savings alone. Further testing with more features will not provide additional insight as the variance drop from additional features would be smaller than the variance drop from adding the consumption feature. This is because each subsequent feature is a poorer predictor of adoption (based on the results in Table $2.2 .^{2}$

\footnotetext{
${ }^{2}$ We also performed principal component analysis (PCA) on the data to asses which components captured greatest variability in the data. PCA corroborated the results of the variance testing and indicated that economic savings and income are the most important factors.
} 


\section{Chapter 3}

\section{Adoption Model}

\subsection{Adoption Model}

Our goal is to create a flexible residential solar PV adoption tool for utility companies and regulators to study the complex relationships between PV adoption and utility economics. Numerous models have been proposed for solar PV adoption [22, 8, 28, 24]. These models range from micro models that are based on representing the likelihood of each individual customer adopting PV to macro models that are based on representing the fraction of the customer base that will adopt PV. We adopt the latter approach as it is likely to require less fitting data and have lower computational complexity. These factors should make our model more accessible to utility companies and regulators and enable them to experiment with multiple scenarios rapidly.

Studies have shown that the prevalence of a new technology has a significant effect on the rate of uptake $[3,4,18,25]$. Moreover, the results of our variance tests indicate the best features for segmenting customers into categories with different likelihoods of adoption. These factors motivate a model where the adoption rate depends on the prevalence and different segments have different adoption rates. For this work, we extend the established Bass diffusion model [24] as it has a simple form that makes it accessible to lay practitioners while still capturing the aggregate impact of different rate schedules and PV incentives. 


\subsubsection{Diffusion model for adoption}

We index time steps by $t \in\{0,1,2, \ldots\} .{ }^{1}$ At each time step $t$, we partition customers into $I$ different segments. We assume that each segment of customers adopts PV based on the current prevalence. Specifically, the probability of adoption in a segment $i \in\{1,2, \ldots, I\}$ is given by the following diffusion model:

$$
\frac{A_{i}[t+1]-A_{i}[t]}{M_{i}[t]}=p_{i}+q_{i}\left(\sum_{i} \frac{A_{i}[t]}{G}\right),
$$

where $A_{i}[t]$ is the number of adopters and $M_{i}[t]$ is the number of customers yet to adopt in segment $i$ at time step $t$. $G$ is the size of the population (adopters and non-adopters) and $p_{i}$ and $q_{i}$ are constant parameters. The constant $p_{i}$ can be interpreted as the coefficient of innovators: customers that adopt PV regardless of the current penetration. The constant $q_{i}$ is the coefficient of imitation: customers that adopt PV based on the fraction of customers who have already adopted.

\subsubsection{Fitting diffusion parameters}

Our variance tests indicate that savings is the best feature for segmenting customers into categories with different likelihoods of adoption. Hence, we partition the range of savings into three categories as defined in the first column of Table 3.1. These partitions are chosen such that there would be a sufficient number of customers in each segment for an accurate fit of $p_{i}$ and $q_{i}$. Note that this gives $M_{i}[t]=G-A_{i}[t]$ which is exactly the classical Bass diffusion model [24].

When fitting diffusion parameters, we restricted our analysis to data after 2008 as there were less that 1500 adopters prior to 2008. To account for customers that are ineligible to install PV due to factors such as shading and renting, we assume that the population size

\footnotetext{
${ }^{1}$ Each step could represent a month or a year. The choice of granularity depends on the available fitting data and desired computational time.
} 
13

\begin{tabular}{llll}
\hline Savings & $p$ & $q$ & RMSE \\
\hline$<\$ 15,000$ & $1 \mathrm{E}-05$ & 0.0113 & 0.9796 \\
$>\$ 15,000 \&<\$ 25,000$ & $5 \mathrm{E}-05$ & 0.0807 & 0.99909 \\
$>\$ 25,000$ & $4 \mathrm{E}-05$ & 0.2127 & 0.99953 \\
\hline
\end{tabular}

Table 3.1: $p, q$ fits for different segmentations.

is $30 \%$ of total number of households in SCE's territory [5]. We calculate $A_{i}[t]$ and $M_{i}[t]$ for each year between 2008 and 2011 and for each bin $i$. Then we fit the following linear function to get values for $p_{i}$ and $q_{i}$ :

$$
Y_{i}=p_{i}+q_{i} X_{i}
$$

where:

$$
Y_{i}=\frac{A_{i}[t+1]-A_{i}[t]}{M_{i}[t]}, \quad X_{i}=\left(\sum_{i} \frac{A_{i}[t]}{G}\right)
$$

The best fit values for $p_{i}$ and $q_{i}$ are shown in Table 3.1.

We observe that, $q_{i}$, the coefficient of imitation increases as potential savings of a customer increase. This indicates the higher the potential savings a customer expects, the more sensitive the customer is to whether the people around him or her has adopted PV. 


\section{Chapter 4}

\section{Simulation Tool}

\subsection{Simulation Tool}

Figure 4.1 gives an overview of the model used to simulate adoption. The diffusion model depends only on customer savings from PV. Hence we categorized customers along the following features: (i) rate schedule, (ii) monthly power consumption, (iii) homeowner/renter. These are the features that affect the adoption savings of customers for any given time step in the simulation. Every year, the utility company revises electricity rates to meet a given revenue requirement. As PV penetration increases, the net usage from customers decrease which leads to higher electricity rates to meet the same revenue requirement.

California recently enacted assembly bill (AB 327) that allows the California Public Utilities Commission (CPUC) to change the utility rate structures as well as the Net Energy Metering (NEM) compensation mechanism [10]. To help inform energy policy decisions, especially in understanding how different rate structures impact solar PV adoption, we built an application using our customer category based model. The application is accessible via the Google App Engine (GAE) at http://etechuptake.appspot.com. 


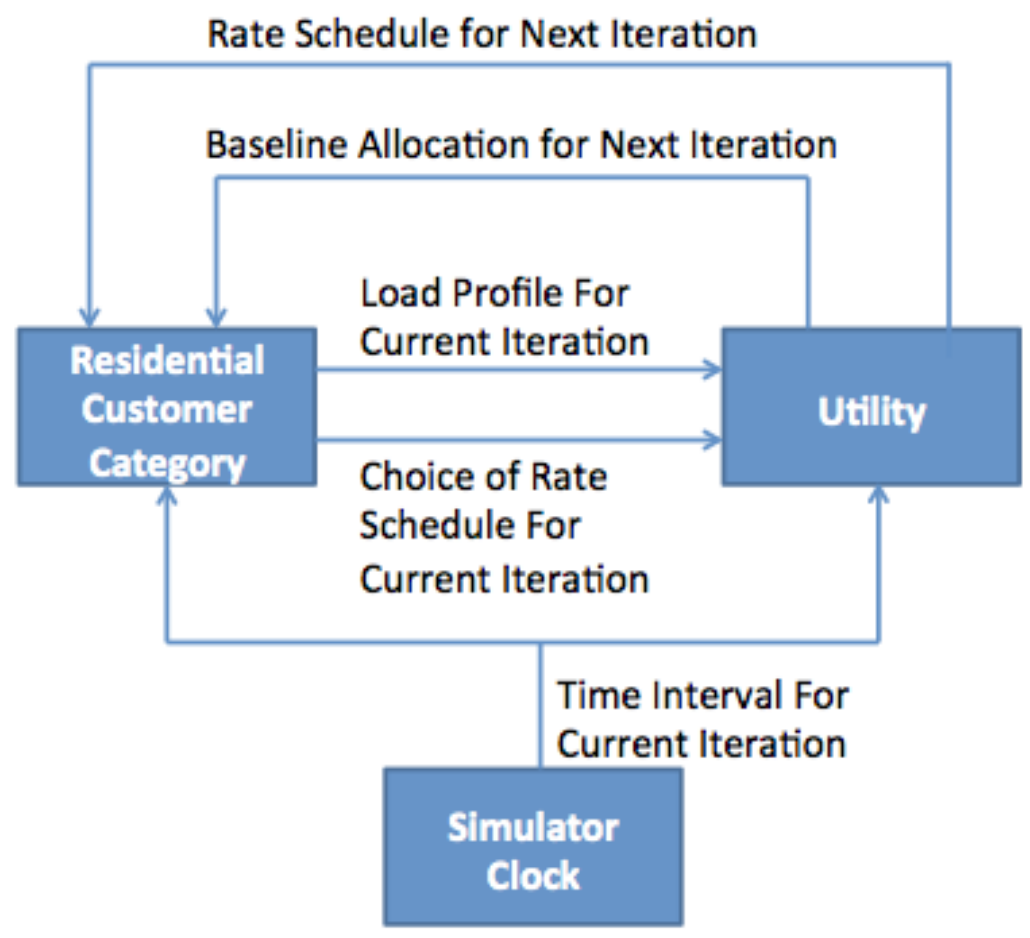

Figure 4.1: Model of PV adoption and rate revision.

\subsection{Simulation Runs}

\subsubsection{Description of different rates and policies tested}

The model results presented in the paper are based on several assumptions. These results are presented merely to show how this and similar diffusion-based models can be used to explore relationships between prices and uptake of technologies. The model results are not predictions. Also, we make no recommendations regarding policy although we hope the model results will encourage use of such models in developing policy.

We run several scenarios to give an idea of how the model can be used. These scenarios explore the following questions: (1) How does number of tiers impact adoption of PV? (2) How do fixed charges impact adoption of PV? (3) How do transitions from multi-tier tariffs 
to fewer tiers impact adoption of PV? (4) What is the impact of tax incentives?

The key features of the scenarios are given in Figure 4.2. Each row describes one scenario. The meanings of the columns are as follows: Tiers: Model Input: Number of tiers in the tariff; Tier 1 Final/Highest Tier Final: Price of the lowest-priced tariff/highest-priced tariff in the final year of the model; Fixed Charge (2015)/(2018): Flat fixed charge all customers pay regardless of consumption in 2015/2018; Rev Req Esc: Percentage increase in revenue requirement of the utility; Change in PV Price: Percentage annual decrease in price of PV.

The first row shows the base case with the current tariff structure. The second row called "PV Drop" shows the impact of a substantial decrease in the cost of PV with prices dropping at $10 \%$ per year as opposed to $5 \%$. The third row, " $4 \mathrm{~T}$ Fixed" shows the impact of introducing a fixed connection charge of $\$ 10$ per month in 2015 for all customers independent of the kWh consumed. The fourth row, "3T Transition", deals with a tariff of only 3 tiers and an introduction of a $\$ 5$ fixed connection charge. The fifth row " $3 \mathrm{~T}$ no Transition", models the same situation without increasing the connection charge in 2015. The sixth row "2T Ratio", uses a different tier structure from the rest of the runs with a fixed ratio between 2 tiers rather than a fixed price difference. The seventh and eighth row, "Q halved" and "P halved", show the sensitivity of the model to the two tuning parameters that were fitted from historical adoption data by halving the $\mathrm{q}$ and $\mathrm{p}$ parameters respectively. The ninth row, "No Revenue Escalation", shows the impact on adoption if we ignore the increase in revenue escalation normally enacted by utilities.

The standard values chosen for different variables are as following, CARE discount is $31 \%$, the utility revenue escalation is $5 \%$, the FTC reduction from $30 \%$ to $10 \%$ in 2017 , the initial cost of $\mathrm{PV}$ is $\$ 5.53 / \mathrm{ACWatt}$ and Tier 1 is initialized to $0.13 \$ / \mathrm{kWh}$. 


\begin{tabular}{|c|c|c|c|c|c|c|c|c|}
\hline Case & $\begin{array}{l}\stackrel{n}{\stackrel{n}{E}} \\
\stackrel{2}{=}\end{array}$ & 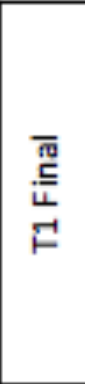 & 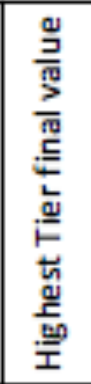 & 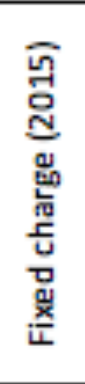 & 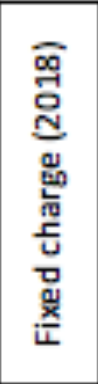 & 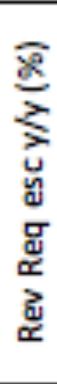 & 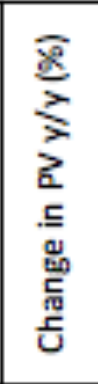 & 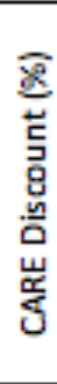 \\
\hline Base & 4 & 0.14 & 0.32 & 0.95 & 1.01 & 5 & $-5 \%$ & 31 \\
\hline PV drop & 4 & 0.14 & 0.33 & 0.95 & 1.01 & 5 & $-10 \%$ & 31 \\
\hline 4T Fixed & 4 & 0.14 & 0.28 & 10 & 10.9 & 5 & $-5 \%$ & 31 \\
\hline 3T Transition & 3 & 0.16 & 0.27 & 5 & 7.5 & 5 & $-5 \%$ & 31 \\
\hline 3T No Transition & 3 & 0.16 & 0.25 & 0.95 & 10.3 & 5 & $-5 \%$ & 31 \\
\hline 2T Ratio w/ Fixed & 2 & 0.18 & 0.3 & 2.5 & 5 & 5 & $-5 \%$ & 31 \\
\hline $\mathrm{Q}$ halved & 4 & 0.14 & 0.32 & 0.95 & 1.01 & 5 & $-5 \%$ & 31 \\
\hline P halved & 4 & 0.14 & 0.32 & 0.95 & 1.01 & 5 & $-5 \%$ & 31 \\
\hline No Rev Escalation & 4 & 0.14 & 0.32 & 0.95 & 1.01 & 0 & $-5 \%$ & 31 \\
\hline
\end{tabular}

Figure 4.2: Description of different rate structures simulated

\subsubsection{Results of simulations}

The results are shown in Figure 4.3. The notable phenomenon are as follows. PV adoption is going to continue to grow but it is likely that the total adoption will plateau with the asymptote depending on model parameters. Secondly, a sharp decrease in PV prices results in a substantial increase in adoption especially among lower-tier customers. Fourth, the three-tier transitional rate produces an adoption curve that is quasi-linear over the next few years. This is in contrast to maintaining four-tier rates but adding a $\$ 10$ fixed charge immediately ("4T Fixed"). Fifth, it appears that the impact of PV prices decreasing at $-10 \%$ per year has an equal magnitude compared to the impact of the expected rate changes in California, although the effects are in opposite directions.

The most dramatic effect on PV adoption is due to the reduction in PV costs. The impact of an annual 10\% decrease in PV prices (compared to the baseline of $5 \%$ ) increases the number of PV adopters by 50\% in October 2018. Hence, financial incentives for PV 


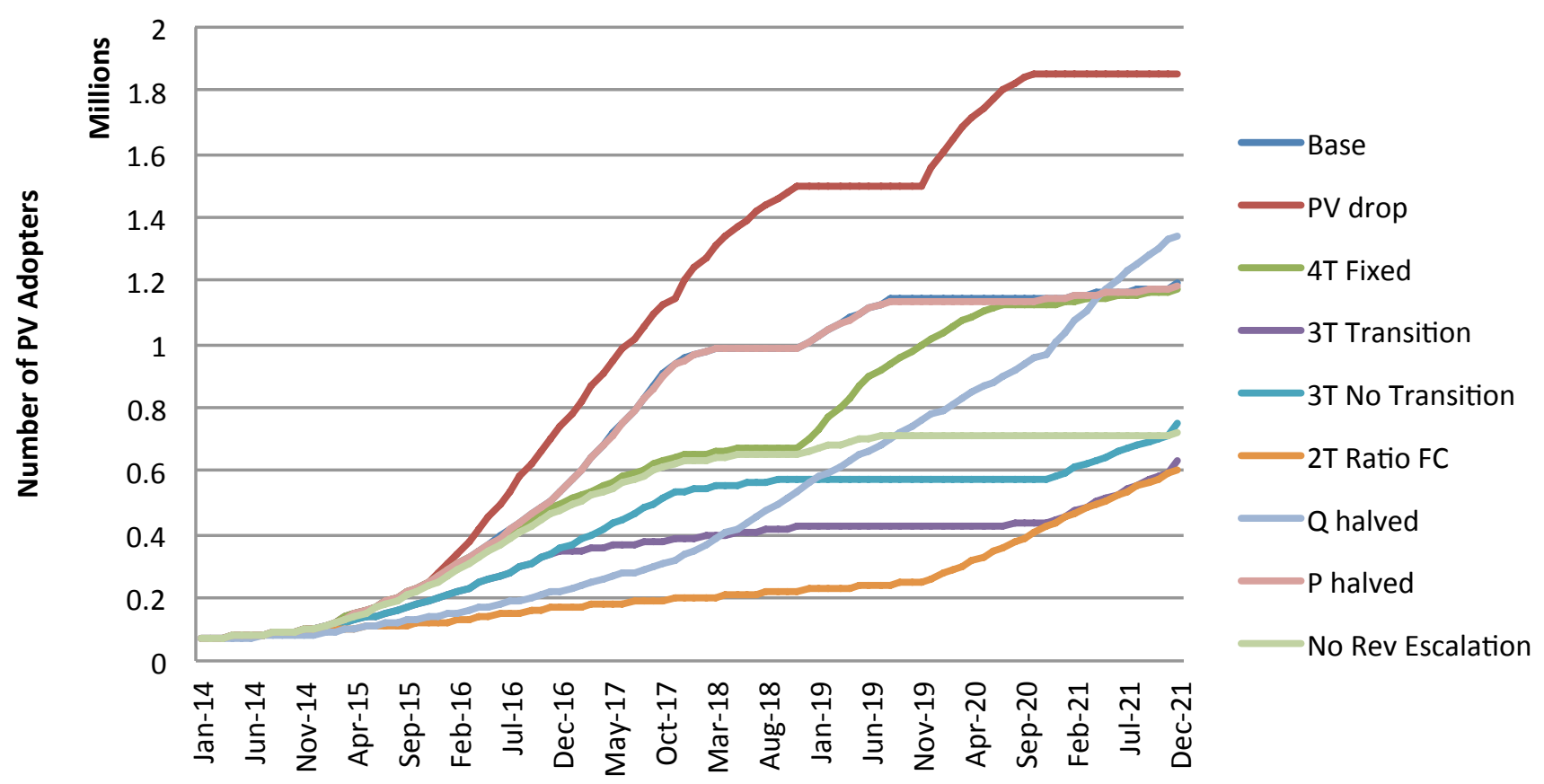

Figure 4.3: Number of adopters over 8 year horizon for different rate structures

have significant capability to disrupt the adoption of PV. When evaluating adoption in Figure 4.3, economic savings due to low cost of PV is a more sensitive factor than rising rates due to decreased consumption.

With regards to the sensitivity of the $\mathrm{p}$, $\mathrm{q}$ fits, we see that halving the $\mathrm{p}$ value (reducing the fraction of innovators by half) has a negligible impact on adoption rate. Halving the $q$ value significantly decreases the rate of adoption of PV. The relatively small fraction of innovators and the strong imitation effect suggests that PV adoption is driven primarily by savings obtained by imitators.

The application offers a technique for users interested in more accurately forecasting month to month adoption numbers. The spikes in adoption are due to bulk shifts of customer categories into new savings bins. A practitioner can use exponential regression to smooth the adoption curve to get a better idea of each timestep's value for the simulation. 
The fitted q value will be based off of the local service territory's historic adoption. 


\section{Chapter 5}

\section{Conclusion}

\subsection{Conclusion}

\subsubsection{Strengths of Model}

The Bass diffusion model variant used in the model which has an embedded savings equation has multiple benefits:

(1) It is fit based on an real, rich data sets from Southern California Edison and robust

public data set offered publicly online by the state of California [9]. By matching this data with energy usage and socioeconomics gives a deeper understanding of what drives customers to adopt PV compared to almost all other PV adoption models. The data set gave the ability to do an in-depth evaluation of multiple dimensions driving customer purchasing behavior and led us to conclude the major driver of adoption is indeed monetary savings.

(2) The second advantage is that the application that was built allows us to rigorously test the sensitivity of different rate structures. This is vitally important in a state such as California where the California Public Utility Commission's (CPUC) initiative to reevaluate residential rate structures [2] has led to transitionary period where new rates are being experimented with, without a firm understanding of the impacts of these changes. The application provided gives users the ability to evaluate changes to fixed charges, num- 
ber of tiers, tier structures and low income assistance rates among others. So this application hopefully goes a long way in providing an avenue to rigorously test the socioeconomic effects of complex changes to energy regulation.

(3) Thirdly by using the Bass diffusion model, a well-established technology adoption equation, the application can capture aggregate customer purchasing tendencies and more importantly, simultaneously model the feedback from lost load due to increased PV adoption. Since the bass diffusion parameter fits are based on historical data, this model would be strong even without incorporating the feedback of loss of load. But by having technology diffusion and feedback in a single model, there finally exists a quantitative model to help answer questions related to the utility "death spiral".

(4) Lastly, the application provides flexibility in inputs beyond simply rate structures. The model computes rates on a year by year bases by first ensuring that utilities have revenue recovery - based on service territory inputs such as total number of customers served, revenue requirement, PV prices and PV production based on individual PV sizes and weather patterns. Hence, this model is applicable to a wide variety of stakeholders including regulators, policymakers, utilities, manufacturers, and universities. By altering the necessary inputs, the impact of adoption can be modeled at a service territory, state or national level.

\subsubsection{Weaknesses of Model}

The model is more sensitive to initial input as the simulation progresses past approximate 5 years - small changes in savings for a customer category can potentially cause large spikes or drops in adoption as the coefficient of imitation in the bass diffusion model is orders of magnitude larger that the coefficient of innovation. Additionally, we only incorporate 24 customer categories based on energy use, type of residence and rate structure. This is sufficient for the use of a utility, but may fall short if state or national level simulations need to made as that would require modeling a prohibitively large number of categories to 
still be accurate.

\subsubsection{Applications}

The intended use of this model is for anyone involved in grid economics to better understand the complex relationship between PV adoption and policy decisions. Utilities can better forecast demand and thus have a more streamlined system planning process. The advantage is that once the system territory is appropriately defined (e.g. number of customers, average sunlight received etc.), the model can be run several times very rapidly with different rate structures and PV prices. Indeed, utilities and policy institutes are already using this tool to simulate how different incentive programs are causing an increase in adoption and in predicting which group of customers will adopt at what specific points in the future.

\subsubsection{Future steps}

The model has been designed in a manner that allows it to be easily extended. A few such extensions would include adding the NEM program changes that will take place on July 1st, 2017[2]. In addition, an upcoming popular rate structure that needs to be added is the Time of Use (TOU) tariff for residential customers. In addition, it would be ideal to add The model also lacks other features that are likely to become important going forward: (1) commercial customers, (2) electric vehicles, and (3) energy storage to have a more comprehensive view of the dynamics involved with the grid going forward. Electric vehicles are only going to increase electricity consumption and so will add to the financial incentive of adopting PV. Modeling the impact of power storage and commercial customers is less clear due to the lack of clarity in the future efficacy of power storage devices and the diversity in the type of customers respectively. 


\section{Bibliography}

$[1]$

[2] R. Benjamin, M. Kito, R. Mutialu, G. Petlin, R. Phillips, and J. Rahman. Energy division staff proposal on residential rate reform. 2014.

[3] B. Bollinger and K. Gillingham. Environmental preferences and peer effects in the diffusion of solar photovoltaic panels. 2010.

[4] B. Bollinger and K. Gillingham. Peer effects in the diffusion of solar photovoltaic panels. Marketing Science, 31(6):900-912, 2012.

[5] D. W. Cai, S. Adlakha, S. H. Low, P. De Martini, and K. Mani Chandy. Impact of residential pv adoption on retail electricity rates. Energy Policy, 62:830-843, 2013.

[6] P. E. Center. California Climate Zones and Bioclimatic Design. http: //www.pge.com/includes/docs/pdfs/about/edusafety/training/pec/ toolbox/arch/climate/californial_climatel_zones।_01-16.pdf ， 2006. (accessed 4/2015).

[7] C. E. Commission. California Building Climate Zones with 2012 Zip Codes. www. energy. ca.gov/maps/renewable/Climatel_Zones।_Zipcode.pdf , 2012. (accessed 5/2014).

[8] A. Créti, J. Joaug, et al. Let the sun shine: Optimal deployment of photovoltaics in germany. 2012. 
[9] CSI. http://www. californiasolarstatistics.ca.gov/ , 2014. (accessed $5 / 2014)$.

[10] N. R. Darghouth, G. Barbose, and R. Wiser. The impact of rate design and net metering on the bill savings from distributed pv for residential customers in california. Energy Policy, 39(9):5243-5253, 2011.

[11] N. R. Darghouth, G. Barbose, and R. H. Wiser. Customer-economics of residential photovoltaic systems (part 1): The impact of high renewable energy penetrations on electricity bill savings with net metering. Energy Policy, 67:290-300, 2014.

[12] DMLSolutions. PRIZM Clusters. http://www. dmlsolutions. com/pdf/Prizm. pdf , 2014. (accessed 5/2014).

[13] E. Drury, M. Miller, C. Macal, D. Graziano, D. Heimiller, J. Ozik, and T. Perry IV. The Transformation of Southern California's Residential Photovoltaics Market through Third-Party Ownership. Energy Policy, 42:681-690, 2012.

[14] V. J. Faden. Net metering of renewable energy: How traditional electricity suppliers fight to keep you in the dark. Widener J. Pub. L., 10:109, 2000.

[15] A. Faiers, M. Cook, and C. Neame. Towards a contemporary approach for understanding consumer behaviour in the context of domestic energy use. Energy Policy, 35(8):4381-4390, 2007.

[16] A. Faiers and C. Neame. Consumer attitudes towards domestic solar power systems. Energy Policy, 34(14):1797-1806, 2006.

[17] A. Ford. System dynamics and the electric power industry. System Dynamics Review, 13(1):57-85, 1997. 
[18] M. Guidolin and C. Mortarino. Cross-country diffusion of photovoltaic systems: modelling choices and forecasts for national adoption patterns. Technological forecasting and social change, 77(2):279-296, 2010.

[19] W. Hoffmann. Pv solar electricity industry: Market growth and perspective. Solar energy materials and solar cells, 90(18):3285-3311, 2006.

[20] A. Ipakchi and F. Albuyeh. Grid of the future. Power and Energy Magazine, IEEE, $7(2): 52-62,2009$.

[21] S. Kalish and G. L. Lilien. A market entry timing model for new technologies. Management Science, 32(2):194-205, 1986.

[22] R. Lobel and G. Perakis. Consumer choice model for forecasting demand and designing incentives for solar technology. Social Science Research Network, MIT, Cambridge, 2011.

[23] Nielsen. Zip Code Look-up. http://www. claritas. com/MyBestSegments/ Default. jsp? ID=20 , 2014. (accessed 5/2014).

[24] J. A. Norton and F. M. Bass. A diffusion theory model of adoption and substitution for successive generations of high-technology products. Management science, 33(9):1069$1086,1987$.

[25] V. Rai and S. A. Robinson. Effective information channels for reducing costs of environmentally-friendly technologies: evidence from residential pv markets. Environmental Research Letters, 8(1):014044, 2013.

[26] V. Rai and B. Sigrin. Diffusion of environmentally-friendly energy technologies: buy versus lease differences in residential pv markets. Environmental Research Letters, 8(1):014022, 2013. 
[27] SCE. Phase 2 of 2012 General Rate Case Rate Design Proposal, SCE/A.1106-07/SCE-04. http://www3. sce. com/sscc/law/dis/dbattach4e.nsf/ O/8FAB7F1E70C260238825792200796804/£FILE/A. 11-06-007\_GRC\+Phasel +2-SCE-04 $\backslash+$ Updated $\backslash+$ Testimony $\backslash$ Redlined $\backslash+$ Version. pdf , 2012a. (accessed $4 / 2015)$.

[28] A. Van Benthem, K. Gillingham, and J. Sweeney. Learning-by-doing and the optimal solar policy in california. The Energy Journal, pages 131-151, 2008.

[29] R. Wallace. The service upgrade. Power and Energy Magazine, IEEE, 8(6):24-27, 2010 . 